\title{
ON THE REPRESENTATION OF MULTI-IDEALS BY TENSOR NORMS
}

\author{
GERALDO BOTELHO ${ }^{\bowtie}$, ERHAN ÇALIŞKAN and DANIEL PELLEGRINO
}

(Received 27 August 2009; accepted 21 September 2010)

Communicated by A. J. Pryde

Abstract

A tensor norm $\beta=\left(\beta_{n}\right)_{n=1}^{\infty}$ is smooth if the natural correspondence

$$
\left(E_{1} \otimes \cdots \otimes E_{n} \otimes \mathbb{K}, \beta_{n+1}\right) \longleftrightarrow\left(E_{1} \otimes \cdots \otimes E_{n}, \beta_{n}\right),
$$

where $\mathbb{K}=\mathbb{R}$ or $\mathbb{C}$, is always an isometric isomorphism. In this paper we study the representation of multi-ideals and of ideals of multilinear forms by smooth tensor norms.

2010 Mathematics subject classification: primary 46G25; secondary 46B28.

Keywords and phrases: multi-ideals, tensor norms.

\section{Introduction and notation}

The idea of describing the dual of a topological tensor product by means of a special class of bilinear mappings goes back to Grothendieck's celebrated 'Résumé' [17]. For example, in his seminal work Grothendieck showed that integral bilinear forms correspond to linear functionals on the injective tensor product. With the emergence of the theory of ideals of multilinear mappings (multi-ideals) between Banach spaces, several instances of this kind of correspondence have appeared. For example, Matos in [19] constructs a tensor norm such that linear operators on the tensor product that are continuous with respect to this norm correspond exactly to the class of nuclear multilinear mappings.

In the theory of multi-ideals, the possibility of moving smoothly from spaces of $(n+1)$-linear mappings down to spaces of $n$-linear mappings turned out to be important (see, for example, [3, 4, 8]). In this note we study tensor norms in which this transition is smooth, as well as multi-ideals that correspond to such smooth tensor norms. To be more precise we need some definitions.

Geraldo Botelho was supported by CNPq Grant 306981/2008-4; Daniel Pellegrino was supported by CNPq Grant 620108/2008-8 (Edital Casadinho) and CNPq Grant 301237/2009-3.

(C) 2011 Australian Mathematical Publishing Association Inc. 1446-7887/2011 \$16.00 
DEFINITION 1.1. An $n$-tensor norm $\beta_{n}$ assigns to every $n$-tuple of normed spaces $E_{1}, \ldots, E_{n}$ a reasonable cross norm $\beta_{n}(\cdot)$ on the full $n$-fold tensor product $E_{1} \otimes$ $\cdots \otimes E_{n}$ that satisfies the metric mapping property. The resulting normed space is denoted by $\left(E_{1} \otimes \cdots \otimes E_{n}, \beta_{n}\right)$. A tensor norm is a sequence $\beta=\left(\beta_{n}\right)_{n=1}^{\infty}$ where each $\beta_{n}$ is an $n$-tensor norm.

Let $E, E_{1}, \ldots, E_{n}, F$ be Banach spaces over the same scalar field $\mathbb{K}$, which is either $\mathbb{R}$ or $\mathbb{C}$. We denote by $\mathcal{L}(E ; F)$ the space of bounded linear operators from $E$ to $F$ endowed with the usual operator norm. When $F=\mathbb{K}$, we simply write $E^{\prime}$. By $\mathcal{L}\left(E_{1}, \ldots, E_{n} ; F\right)$ we mean the space of continuous $n$-linear mappings from $E_{1} \times \cdots \times E_{n}$ to $F$ endowed with the usual supremum norm. When $E_{1}=\cdots=$ $E_{n}=E$ we write $\mathcal{L}\left({ }^{n} E ; F\right)$.

Definition 1.2. An ideal of multilinear mappings (or multi-ideal) $\mathcal{M}$ is a subclass of the class of all continuous multilinear mappings between Banach spaces such that, given a positive integer $n$, and Banach spaces $E_{1}, \ldots, E_{n}$ and $F$, the components $\mathcal{M}\left(E_{1}, \ldots, E_{n} ; F\right)$, defined to be $\mathcal{L}\left(E_{1}, \ldots, E_{n} ; F\right) \cap \mathcal{M}$, satisfy the following conditions.

(i) $\mathcal{M}\left(E_{1}, \ldots, E_{n} ; F\right)$ is a linear subspace of $\mathcal{L}\left(E_{1}, \ldots, E_{n} ; F\right)$ that contains the $n$-linear mappings of finite type.

(ii) The ideal property: if $A \in \mathcal{M}\left(E_{1}, \ldots, E_{n} ; F\right), u_{j} \in \mathcal{L}\left(G_{j} ; E_{j}\right)$ when $j=$ $1, \ldots, n$ and $t \in \mathcal{L}(F ; H)$, then

$$
t \circ A \circ\left(u_{1}, \ldots, u_{n}\right) \in \mathcal{M}\left(G_{1}, \ldots, G_{n} ; H\right) .
$$

Moreover, there is a function $\|\cdot\|_{\mathcal{M}}: \mathcal{M} \longrightarrow \mathbb{R}^{+}$with the following properties.

(iii) $\|\cdot\|_{\mathcal{M}}$ restricted to $\mathcal{M}\left(E_{1}, \ldots, E_{n} ; F\right)$ is a norm for all Banach spaces $E_{1}, \ldots, E_{n}$ and $F$ that makes $\mathcal{M}\left(E_{1}, \ldots, E_{n} ; F\right)$ a Banach space.

(iv) If $A: \mathbb{K}^{n} \longrightarrow \mathbb{K}$ is defined by $A\left(\lambda_{1}, \ldots, \lambda_{n}\right)=\lambda_{1} \cdots \lambda_{n}$, then $\|A\|_{\mathcal{M}}=1$, for all $n$.

(v) If $A \in \mathcal{M}\left(E_{1}, \ldots, E_{n} ; F\right), u_{j} \in \mathcal{L}\left(G_{j} ; E_{j}\right)$ when $j=1, \ldots, n$ and $t \in$ $\mathcal{L}(F ; H)$, then

$$
\left\|t \circ A \circ\left(u_{1}, \ldots, u_{n}\right)\right\|_{\mathcal{M}} \leq\|t\|\|A\|_{\mathcal{M}}\left\|u_{1}\right\| \cdots\left\|u_{n}\right\| .
$$

Of course the Banach spaces considered in this definition are all over the same fixed scalar field. We define

$$
\mathcal{M}^{\mathbb{K}}:=\left\{\mathcal{M}\left(E_{1}, \ldots, E_{n} ; \mathbb{K}\right): n \in \mathbb{N} \text { and } E_{1}, \ldots, E_{n} \text { are Banach spaces }\right\},
$$

and say that $\mathcal{M}^{\mathbb{K}}$ is an ideal of multilinear forms.

DEFINITION 1.3. We say that a tensor norm $\beta=\left(\beta_{n}\right)_{n=1}^{\infty}$ represents the multi-ideal $\mathcal{M}$, or $\mathcal{M}$ is $\beta$-represented, if $\mathcal{M}\left(E_{1}, \ldots, E_{n} ; F^{\prime}\right)$ and $\left(E_{1} \otimes \cdots \otimes E_{n} \otimes F, \beta_{n+1}\right)^{\prime}$ are isometrically isomorphic under the canonical mapping

$$
\begin{gathered}
\varphi: \mathcal{M}\left(E_{1}, \ldots, E_{n} ; F^{\prime}\right) \longrightarrow\left(E_{1} \otimes \cdots \otimes E_{n} \otimes F, \beta_{n+1}\right)^{\prime} \\
T \mapsto \varphi(T)\left(x_{1} \otimes \cdots \otimes x_{n} \otimes y\right)=T\left(x_{1}, \ldots, x_{n}\right)(y),
\end{gathered}
$$

for all $n$ and all Banach spaces $E_{1}, \ldots, E_{n}, F$. 
The ideal of multilinear forms $\mathcal{M}^{\mathbb{K}}$ is represented by $\beta$, or $\mathcal{M}^{\mathbb{K}}$ is $\beta$-represented, if the condition above holds for all components $\mathcal{M}\left(E_{1}, \ldots, E_{n} ; \mathbb{K}\right)$ of $\mathcal{M}^{\mathbb{K}}$.

In [16, Theorem 4.5] it is proved that a multi-ideal $\mathcal{M}$ is maximal if and only if $\mathcal{M}$ is represented by some (finitely generated) tensor norm. It is well known (see, for example, [11, Exercise 12.1]) that $(E \otimes \mathbb{K}, \alpha)$ is isometrically isomorphic to $E$ via the correspondence $x \otimes \lambda \longleftrightarrow \lambda x$ for all normed spaces $E$ and 2-tensor norms $\alpha$. Given a tensor norm $\beta=\left(\beta_{n}\right)_{n=1}^{\infty}$, this property can be rewritten as $\left(E \otimes \mathbb{K}, \beta_{2}\right)=\left(E, \beta_{1}\right)$ for all $E$. As we will see, this property is not valid for larger $n$, that is, it is not always true that $\left(E_{1} \otimes \cdots \otimes E_{n}, \beta_{n}\right)$ is canonically isomorphic to $\left(E_{1} \otimes \cdots \otimes E_{n} \otimes \mathbb{K}, \beta_{n+1}\right)$ for all $n \geq 2$. This phenomenon motivates the following definition.

DEFINITION 1.4. A tensor norm $\beta=\left(\beta_{n}\right)_{n=1}^{\infty}$ is said to be smooth if, for all natural $n$ and all normed spaces $E_{1}, \ldots, E_{n}$, the natural map

$$
\begin{gathered}
\psi:\left(E_{1} \otimes \cdots \otimes E_{n} \otimes \mathbb{K}, \beta_{n+1}\right) \longrightarrow\left(E_{1} \otimes \cdots \otimes E_{n}, \beta_{n}\right), \\
\psi\left(x_{1} \otimes \cdots \otimes x_{n} \otimes \lambda\right)=\lambda\left(x_{1} \otimes \cdots \otimes x_{n}\right)
\end{gathered}
$$

is an isometric isomorphism.

In this paper we are concerned with the representation of multi-ideals by smooth tensor norms. The conclusion of our results and examples is that multi-ideals are rarely represented by smooth tensor norms, that ideals of multilinear forms are more often represented by smooth tensor norms, and that the representation of an ideal of multilinear forms by a smooth tensor norm yields the representation of some of its vector-valued components by the same smooth tensor norm.

\section{The vector-valued case}

The aim of this section is to show that multi-ideals, including their vector-valued components, are rarely represented by smooth tensor norms. We start with two obvious examples.

EXAMPLE 2.1. The projective tensor norm $\pi$ is smooth: given

$$
z=\sum_{j=1}^{m} x_{j}^{(1)} \otimes \cdots \otimes x_{j}^{(n)} \otimes \lambda_{j} \in E_{1} \otimes \cdots \otimes E_{n} \otimes \mathbb{K}
$$

we define

$$
\bar{z}=\sum_{j=1}^{m} \lambda_{j} x_{j}^{(1)} \otimes \cdots \otimes x_{j}^{(n)} \in E_{1} \otimes \cdots \otimes E_{n},
$$

and can easily check that $\pi_{n+1}(z)=\pi_{n}(\bar{z})$. So

$$
\psi:\left(E_{1} \otimes \cdots \otimes E_{n} \otimes \mathbb{K}, \pi_{n+1}\right) \longrightarrow\left(E_{1} \otimes \cdots \otimes E_{n}, \pi_{n}\right)
$$

is an isometric isomorphism. So the multi-ideal $\mathcal{L}$ of all continuous multilinear mappings between Banach spaces, which is obviously $\pi$-represented (see [10, Proposition A.3.7]), is represented by a smooth tensor norm. 
EXAMPLE 2.2. The injective tensor norm $\varepsilon$ is smooth. Indeed, for $z$ and $\bar{z}$ as above,

$$
\begin{aligned}
\varepsilon_{n+1}(z) & =\sup _{\varphi l \in B_{E_{l}^{\prime}}, \varphi \in B_{\mathbb{K}^{\prime}}}\left|\sum_{j=1}^{m} \varphi_{1}\left(x_{j}^{(1)}\right) \cdots \varphi_{n}\left(x_{j}^{(n)}\right) \varphi\left(\lambda_{j}\right)\right| \\
& =\sup _{\varphi_{l} \in B_{E_{l}^{\prime}}, \varphi \in B_{\mathbb{K}^{\prime}}}\left|\sum_{j=1}^{m} \varphi_{1}\left(\lambda_{j} x_{j}^{(1)}\right) \cdots \varphi_{n}\left(x_{j}^{(n)}\right) \varphi(1)\right| \\
& =\sup _{\varphi l \in B_{E_{l}^{\prime}}, \varphi \in B_{\mathbb{K}^{\prime}}}|\varphi(1)|\left|\sum_{j=1}^{m} \varphi_{1}\left(\lambda_{j} x_{j}^{(1)}\right) \cdots \varphi_{n}\left(x_{j}^{(n)}\right)\right| \\
& =\sup _{\varphi_{l} \in B_{E_{l}^{\prime}}}\left|\sum_{j=1}^{m} \varphi_{1}\left(\lambda_{j} x_{j}^{(1)}\right) \cdots \varphi_{n}\left(x_{j}^{(n)}\right)\right|=\varepsilon_{n}(\widetilde{z}),
\end{aligned}
$$

which proves that

$$
\psi:\left(E_{1} \otimes \cdots \otimes E_{n} \otimes \mathbb{K}, \varepsilon_{n+1}\right) \longrightarrow\left(E_{1} \otimes \cdots \otimes E_{n}, \varepsilon_{n}\right)
$$

is an isometric isomorphism. It has been known since Grothendieck's 'Résumé' that the multi-ideal $\mathcal{L}_{\mathcal{I}}$ of integral multilinear mappings is $\varepsilon$-represented (the scalar-valued bilinear case can be found in [13, Theorem 1.1.21]). We give the details for the sake of completeness. Denoting by $\mathcal{I}(E ; F)$ the space of integral linear operators from $E$ to $F$ and by $E_{1} \widehat{\otimes}_{\varepsilon} \cdots \widehat{\otimes}_{\varepsilon} E_{n}$ the completion of $\left(E_{1} \otimes \cdots \otimes E_{n}, \varepsilon_{n}\right)$, we see that

$$
\begin{aligned}
\mathcal{L}_{\mathcal{I}}\left(E_{1}, \ldots, E_{n} ; F^{\prime}\right) & =\mathcal{I}\left(E_{1} \widehat{\otimes}_{\varepsilon} \cdots \widehat{\otimes}_{\varepsilon} E_{n} ; F^{\prime}\right) \\
& =\mathcal{L}_{\mathcal{I}}\left(E_{1} \widehat{\otimes}_{\varepsilon} \cdots \widehat{\otimes}_{\varepsilon} E_{n}, F ; \mathbb{K}\right) \\
& =\mathcal{I}\left(E_{1} \widehat{\otimes}_{\varepsilon} \cdots \widehat{\otimes}_{\varepsilon} E_{n} \widehat{\otimes}_{\varepsilon} F ; \mathbb{K}\right) \\
& =\left(E_{1} \widehat{\otimes}_{\varepsilon} \cdots \widehat{\otimes}_{\varepsilon} E_{n} \widehat{\otimes}_{\varepsilon} F\right)^{\prime} \\
& =\left(E_{1} \otimes \cdots \otimes E_{n} \otimes F, \varepsilon_{n+1}\right)^{\prime} .
\end{aligned}
$$

For a proof of the first and third equalities, see [9, Proposition 2.2]; for the second equality, see [22, Proposition 3.22]; the fourth equality is trivial because $\mathcal{I}$ is an operator ideal. For the last equality, observe that since $E_{1} \widehat{\otimes}_{\varepsilon} \cdots \widehat{\otimes}_{\varepsilon} E_{n} \widehat{\otimes}_{\varepsilon} F$ is the completion of the normed space $\left(E_{1} \otimes \cdots \otimes E_{n} \otimes F, \varepsilon_{n+1}\right)$, we can identify the space $\left(E_{1} \otimes \cdots \otimes E_{n} \otimes F, \varepsilon_{n+1}\right)^{\prime}$ with $\left(E_{1} \widehat{\otimes}_{\varepsilon} \cdots \widehat{\otimes}_{\varepsilon} E_{n} \widehat{\otimes}_{\varepsilon} F\right)^{\prime}$ through the familiar isometry $\varphi \longrightarrow \bar{\varphi}$, where $\bar{\varphi}$ is the unique extension of $\varphi$.

Since all identifications above are by the corresponding standard mappings, their composition coincides with our mapping $\varphi$, which proves that $\mathcal{L}_{\mathcal{I}}$ is $\varepsilon$-represented, hence represented by a smooth tensor norm.

Definition 2.3. Let $\beta=\left(\beta_{n}\right)_{n=1}^{\infty}$ be a tensor norm. Define $\mathcal{L}_{\beta}\left(E_{1}, \ldots, E_{n} ; F\right)$ to be the set of those multilinear mappings $A \in \mathcal{L}\left(E_{1}, \ldots, E_{n} ; F\right)$ whose linearizations

$$
A_{L}:\left(E_{1} \otimes \cdots \otimes E_{n}, \beta_{n}\right) \longrightarrow F
$$


are continuous, endowed with the norm

$$
\|A\|_{\mathcal{L}_{\beta}}:=\left\|A_{L}:\left(E_{1} \otimes \cdots \otimes E_{n}, \beta_{n}\right) \longrightarrow F\right\| .
$$

It is easy to see that $\mathcal{L}_{\beta}$ is a multi-ideal. Whether or not $\mathcal{L}_{\beta}$ is $\beta$-represented is a quite natural question. We shall treat it later.

Next we define a property that is closely related to property (B) of [3].

Definition 2.4. Given $A \in \mathcal{L}\left(E_{1}, \ldots, E_{n}, \mathbb{K} ; F\right)$, define $A 1 \in \mathcal{L}\left(E_{1}, \ldots, E_{n} ; F\right)$ by

$$
A 1\left(x_{1}, \ldots, x_{n}\right)=A\left(x_{1}, \ldots, x_{n}, 1\right) .
$$

We say that a multi-ideal $\mathcal{M}$ has property $[\mathrm{B}]$ if

$$
A \in \mathcal{M}\left(E_{1}, \ldots, E_{n}, \mathbb{K} ; F\right) \Longleftrightarrow A 1 \in \mathcal{M}\left(E_{1}, \ldots, E_{n} ; F\right),
$$

and in this case $\|A\|_{\mathcal{M}}=\|A 1\|_{\mathcal{M}}$ for all $n$, for all spaces $E_{1}, \ldots, E_{n}, F$ and for all maps $A \in \mathcal{L}\left(E_{1}, \ldots, E_{n} ; F\right)$.

PROPOSITION 2.5. A tensor norm $\beta$ is smooth if and only if its corresponding multiideal $\mathcal{L}_{\beta}$ has property [B].

PROOF. We begin by assuming that $\beta=\left(\beta_{n}\right)_{n=1}^{\infty}$ is a smooth tensor norm. Given $A \in \mathcal{L}\left(E_{1}, \ldots, E_{n}, \mathbb{K} ; F\right)$, consider the chain

$$
E_{1} \otimes \cdots \otimes E_{n} \otimes \mathbb{K} \stackrel{\psi}{\longrightarrow} E_{1} \otimes \cdots \otimes E_{n} \stackrel{\psi^{-1}}{\longrightarrow} E_{1} \otimes \cdots \otimes E_{n} \otimes \mathbb{K} \stackrel{A_{L}}{\longrightarrow} F .
$$

It is not difficult to see that $A_{L}=(A 1)_{L} \circ \psi$ and $(A 1)_{L}=A_{L} \circ \psi^{-1}$. Since $\psi$ and $\psi^{-1}$ are continuous as $\beta$ is smooth, it follows that $A_{L}$ is continuous if and only if $(A 1)_{L}$ is continuous, that is, $A \in \mathcal{L}_{\beta}$ if and only if $A 1 \in \mathcal{L}_{\beta}$. In this case $\|A\|_{\mathcal{L}_{\beta}}=\|A 1\|_{\mathcal{L}_{\beta}}$ because $\psi$ and $\psi^{-1}$ are isometric isomorphisms, proving that $\mathcal{L}_{\beta}$ has property $[\mathrm{B}]$.

Conversely, assume that $\mathcal{L}_{\beta}$ has property [B]. Given $E_{1}, \ldots, E_{n}$, define

$$
A: E_{1} \times \cdots \times E_{n} \times \mathbb{K} \longrightarrow\left(E_{1} \otimes \cdots \otimes E_{n}, \beta_{n}\right),
$$

by $A\left(x_{1}, \ldots, x_{n}, \lambda\right)=\lambda\left(x_{1} \otimes \cdots \otimes x_{n}\right)$. Then

$$
(A 1)_{L}\left(x_{1} \otimes \cdots \otimes x_{n}\right)=A 1\left(x_{1}, \ldots, x_{n}\right)=A\left(x_{1}, \ldots, x_{n}, 1\right)=x_{1} \otimes \cdots \otimes x_{n},
$$

showing that $(A 1)_{L}$ is the identity operator on $E_{1} \otimes \cdots \otimes E_{n}$, and hence is an isometric isomorphism when this space is endowed with $\beta_{n}$ on both sides. It follows that

$$
A 1 \in \mathcal{L}_{\beta}\left(E_{1}, \ldots, E_{n} ;\left(E_{1} \otimes \cdots \otimes E_{n}, \beta_{n}\right)\right) .
$$

So $A \in \mathcal{L}_{\beta}\left(E_{1}, \ldots, E_{n}, \mathbb{K} ;\left(E_{1} \otimes \cdots \otimes E_{n}, \beta_{n}\right)\right)$ and

$$
\begin{aligned}
\|A\|_{\mathcal{L}_{\beta}}=\|A 1\|_{\mathcal{L}_{\beta}} & =\|(A 1)_{L}:\left(E_{1} \otimes \cdots \otimes E_{n}, \beta_{n}\right) \\
& \longrightarrow\left(E_{1} \otimes \cdots \otimes E_{n}, \beta_{n}\right) \|=1
\end{aligned}
$$


as $\mathcal{L}_{\beta}$ has property [B]. Therefore the map

$$
A_{L}:\left(E_{1} \otimes \cdots \otimes E_{n} \otimes \mathbb{K}, \beta_{n+1}\right) \longrightarrow\left(E_{1} \otimes \cdots \otimes E_{n}, \beta_{n}\right)
$$

is continuous. But $A_{L}=\psi$, so $\psi$ is continuous and

$$
1=\|A\|_{\mathcal{L}_{\beta}}=\left\|A_{L}:\left(E_{1} \otimes \cdots \otimes E_{n} \otimes \mathbb{K}, \beta_{n+1}\right) \longrightarrow\left(E_{1} \otimes \cdots \otimes E_{n}, \beta_{n}\right)\right\| .
$$

Moreover,

$$
\beta_{n}(\psi(z))=\beta_{n}\left(A_{L}(z)\right) \leq \beta_{n+1}(z)
$$

for all $z \in E_{1} \otimes \cdots \otimes E_{n} \otimes \mathbb{K}$.

Consider now the map

$$
\begin{gathered}
C: E_{1} \times \cdots \times E_{n} \times \mathbb{K} \longrightarrow\left(E_{1} \otimes \cdots \otimes E_{n} \otimes \mathbb{K}, \beta_{n+1}\right) \\
C\left(x_{1}, \ldots, x_{n}, \lambda\right)=x_{1} \otimes \cdots \otimes x_{n} \otimes \lambda
\end{gathered}
$$

It is clear that $C_{L}$ is the identity operator on $E_{1} \otimes \cdots \otimes E_{n} \otimes \mathbb{K}$, hence is an isometric isomorphism when this space is endowed with $\beta_{n+1}$ on both sides. Thus

$$
C \in \mathcal{L}_{\beta}\left(E_{1}, \ldots, E_{n}, \mathbb{K} ;\left(E_{1} \otimes \cdots \otimes E_{n} \otimes \mathbb{K}, \beta_{n+1}\right)\right) .
$$

So $C 1 \in \mathcal{L}_{\beta}\left(E_{1}, \ldots, E_{n} ;\left(E_{1} \otimes \cdots \otimes E_{n} \otimes \mathbb{K}, \beta_{n+1}\right)\right)$ and

$$
\begin{gathered}
\|C 1\|_{\mathcal{L}_{\beta}}=\|C\|_{\mathcal{L}_{\beta}}=\| C_{L}:\left(E_{1} \otimes \cdots \otimes E_{n} \otimes \mathbb{K}, \beta_{n+1}\right) \\
\longrightarrow\left(E_{1} \otimes \cdots \otimes E_{n} \otimes \mathbb{K}, \beta_{n+1}\right) \|=1
\end{gathered}
$$

as $\mathcal{L}_{\beta}$ has property [B]. Therefore the map

$$
(C 1)_{L}:\left(E_{1} \otimes \cdots \otimes E_{n}, \beta_{n}\right) \longrightarrow\left(E_{1} \otimes \cdots \otimes E_{n} \otimes \mathbb{K}, \beta_{n+1}\right)
$$

is continuous. But $(C 1)_{L}=\psi^{-1}$, so $\psi^{-1}$ is continuous and

$$
1=\|C 1\|_{\mathcal{L}_{\beta}}=\left\|(C 1)_{L}:\left(E_{1} \otimes \cdots \otimes E_{n}, \beta_{n}\right) \longrightarrow\left(E_{1} \otimes \cdots \otimes E_{n} \times \mathbb{K}, \beta_{n+1}\right)\right\| .
$$

Moreover,

$$
\beta_{n+1}\left(\psi^{-1}(w)\right)=\beta_{n+1}\left((C 1)_{L}(w)\right) \leq \beta_{n}(w)
$$

for all $w \in E_{1} \otimes \cdots \otimes E_{n}$. Taking $w=\psi(z)$, we obtain

$$
\beta_{n}(\psi(z)) \leq \beta_{n+1}(z) \leq \beta_{n}(\psi(z))
$$

for all $z \in E_{1} \otimes \cdots \otimes E_{n} \otimes \mathbb{K}$, proving that $\psi$ is an isometric isomorphism, that is, $\beta$ is smooth.

We now turn our attention to multi-ideals that can be represented by smooth tensor norms. 
THEOREM 2.6 (Uniqueness of the representation). The tensor norm that represents $a$ given multi-ideal, if any, is unique.

PROOF. Let $\mathcal{M}$ be a multi-ideal that is represented by the tensor norms $\beta=\left(\beta_{n}\right)_{n=1}^{\infty}$ and $\gamma=\left(\gamma_{n}\right)_{n=1}^{\infty}$. Let $E_{1}, \ldots, E_{n-1}, E_{n}$ be given. The corresponding operators

$$
\varphi_{\beta}: \mathcal{M}\left(E_{1}, \ldots, E_{n-1} ; E_{n}^{\prime}\right) \longrightarrow\left(E_{1} \otimes \cdots \otimes E_{n}, \beta_{n}\right)^{\prime}
$$

and

$$
\varphi_{\gamma}: \mathcal{M}\left(E_{1}, \ldots, E_{n-1} ; E_{n}^{\prime}\right) \longrightarrow\left(E_{1} \otimes \cdots \otimes E_{n}, \gamma_{n}\right)^{\prime}
$$

are isometric isomorphisms. So the composition $\varphi_{\beta} \circ\left(\varphi_{\gamma}\right)^{-1}$, which is clearly the formal identity, is an isometric isomorphism from $\left(E_{1} \otimes \cdots \otimes E_{n}, \gamma_{n}\right)^{\prime}$ to $\left(E_{1} \otimes \cdots \otimes E_{n}, \beta_{n}\right)^{\prime}$. By the Hahn-Banach theorem, it follows that $\gamma_{n}=\beta_{n}$ on $E_{1} \otimes \cdots \otimes E_{n}$.

We have already mentioned that [16, Theorem 4.5] ensures the existence of a tensor norm that represents $\mathcal{M}$, given a maximal multi-ideal $\mathcal{M}$ (for the definition see [16]). Let us denote this tensor norm, which is unique by Theorem 2.6, by $\beta^{\mathcal{M}}$. Combining Proposition 2.5 and Theorem 2.6, we obtain the following result.

PROPOSITION 2.7. The following are equivalent for a maximal multi-ideal $\mathcal{M}$.

(a) $\mathcal{M}$ is represented by a smooth tensor norm.

(b) $\beta^{\mathcal{M}}$ is smooth.

(c) $\mathcal{L}_{\beta^{\mathcal{M}}}$ has property [B].

In particular, if $\mathcal{M}$ has property [B] and $\mathcal{M}=\mathcal{L}_{\beta \mathcal{M}}$, then $\mathcal{M}$ is represented by a smooth tensor norm.

This result impels us to study the equality $\mathcal{M}=\mathcal{L}_{\beta} \mathcal{M}$. As to the projective norm, by Example 2.1 and Theorem 2.6, we know that $\beta^{\mathcal{L}}=\pi$, so $\mathcal{L}_{\beta} \mathcal{L}=\mathcal{L}_{\pi}=\mathcal{L}$. We treat this question together with the question of whether $\mathcal{L}_{\beta}$ is $\beta$-represented.

PROPOSITION 2.8. The multi-ideal $\mathcal{L}_{\varepsilon}$ is not $\varepsilon$-represented.

ProOf. Assume for the moment that $\mathcal{L}_{\varepsilon}$ is $\varepsilon$-represented. On the one hand, by Example 2.2, $\mathcal{L}_{\mathcal{I}}$ is $\varepsilon$-represented, and it follows easily that

$$
\mathcal{L}_{\varepsilon}\left(E_{1}, \ldots, E_{n} ; F^{\prime}\right)=\mathcal{L}_{\mathcal{I}}\left(E_{1}, \ldots, E_{n} ; F^{\prime}\right)
$$

for all $n$ and $E_{1}, \ldots, E_{n}, F$. On the other hand, by Example 2.2 and Theorem 2.6, we know that $\beta^{\mathcal{L}_{\mathcal{I}}}=\varepsilon$ and from [9, Proposition 2.2], $\mathcal{L}_{\mathcal{I}} \subseteq \mathcal{L}_{\varepsilon}$, hence $\mathcal{L}_{\mathcal{I}} \subseteq \mathcal{L}_{\varepsilon}=\mathcal{L}_{\beta} \mathcal{L}_{\mathcal{I}}$.

As to the converse inclusion, let $n \geq 2$ and $F$ be an infinite-dimensional Banach space. Assume for the moment that $\mathcal{L}\left(\hat{\bigotimes}_{n, \varepsilon} c_{0} ; F\right)=\mathcal{I}\left(\hat{\bigotimes}_{n, \varepsilon} c_{0} ; F\right)$. As integral linear operators are absolutely summing [14, Proposition 5.5], it follows that every continuous linear operator from $\hat{\bigotimes}_{n, \varepsilon} c_{0}$ to $F$ is absolutely summing. We know that $\hat{\bigotimes}_{n, \varepsilon} c_{0}$ has an unconditional basis because $c_{0}=\hat{\bigotimes}_{n, \varepsilon} c_{0}$, so by a result of Lindenstrauss and Pełczyński [18, Theorem 4.2], it follows that $\hat{\bigotimes}_{n, \varepsilon} c_{0}=c_{0}$ is isomorphic to some $\ell_{1}(\Gamma)$, but this is absurd. Therefore there exists a nonintegral 
operator $u \in \mathcal{L}\left(\hat{\bigotimes}_{n, \varepsilon} c_{0} ; F\right)$. Define

$$
A: c_{0} \times \cdots \times c_{0} \longrightarrow F, \quad A\left(x_{1}, \ldots, x_{n}\right)=u\left(x_{1} \otimes \cdots \otimes x_{n}\right) .
$$

So $A_{L}=u$ is $\varepsilon$-continuous, hence $A \in \mathcal{L}_{\varepsilon}\left({ }^{n} c_{0} ; F\right)$, but $A$ fails to be integral because its linearization $A_{L}=u$ is not integral in the injective norm $\varepsilon$. Hence

$$
\mathcal{L}_{\beta} \mathcal{L}_{\mathcal{I}}\left({ }^{n} c_{0} ; F\right)=\mathcal{L}_{\varepsilon}\left({ }^{n} c_{0} ; F\right) \neq \mathcal{L}_{\mathcal{I}}\left({ }^{n} c_{0} ; F\right),
$$

which is a contradiction.

Later, in Proposition 2.17, we shall go further. For the moment, the proof above shows, in particular, that $\mathcal{L}_{\beta} \mathcal{L}_{\mathcal{I}} \neq \mathcal{L}_{\mathcal{I}}$. Corollary 2.12 below provides another example of the inequality $\mathcal{M} \neq \mathcal{L}_{\beta} \mathcal{M}$.

Now we proceed to present some multi-ideals that are represented by tensor norms but not by smooth tensor norms. First, we give a general criterion.

Proposition 2.9. Let $\mathcal{M}$ be a multi-ideal such that

$$
\mathcal{M}\left(E_{1}, \ldots, E_{n}, F ; \mathbb{K}\right)=\mathcal{L}\left(E_{1}, \ldots, E_{n}, F ; \mathbb{K}\right)
$$

and

$$
\mathcal{M}\left(E_{1}, \ldots, E_{n} ; F^{\prime}\right) \neq \mathcal{L}\left(E_{1}, \ldots, E_{n} ; F^{\prime}\right)
$$

for some Banach spaces $E_{1}, \ldots, E_{n}, F$ and some positive integer $n$. Then there is no smooth tensor norm that represents $\mathcal{M}$.

PROOF. Assume that there is a smooth tensor norm $\beta=\left(\beta_{n}\right)_{n=1}^{\infty}$ that represents $\mathcal{M}$. Since $\beta$ is smooth, the adjoint $\psi^{*}$ of $\psi$ is an isometric isomorphism from the space $\left(E_{1} \otimes \cdots \otimes E_{n} \otimes F, \beta_{n+1}\right)^{\prime}$ to $\left(E_{1} \otimes \cdots \otimes E_{n} \otimes F \otimes \mathbb{K}, \beta_{n+2}\right)^{\prime}$. We have the following chain of isomorphisms:

$$
\begin{aligned}
\left(E_{1} \otimes \cdots \otimes E_{n} \otimes F, \beta_{n+1}\right)^{\prime} & \stackrel{\psi^{*}}{\longleftrightarrow}\left(E_{1} \otimes \cdots \otimes E_{n} \otimes F \otimes \mathbb{K}, \beta_{n+2}\right)^{\prime} \\
& \stackrel{\varphi}{\longleftrightarrow} \mathcal{M}\left(E_{1}, \ldots, E_{n}, F ; \mathbb{K}^{\prime}\right) \\
& \longleftrightarrow \mathcal{M}\left(E_{1}, \ldots, E_{n}, F ; \mathbb{K}\right) \\
& \stackrel{\text { id }}{\longleftrightarrow}\left(E_{1}, \ldots, E_{n}, F ; \mathbb{K}\right) \\
& \longleftrightarrow\left(E_{1} \otimes \cdots \otimes E_{n} \otimes F, \pi_{n+1}\right)^{\prime},
\end{aligned}
$$

where the mappings that are not labeled are canonical. It follows that the identity operator is an algebraic isomorphism between the spaces $\left(E_{1} \otimes \cdots \otimes E_{n} \otimes F, \beta_{n+1}\right)^{\prime}$ and $\left(E_{1} \otimes \cdots \otimes E_{n} \otimes F, \pi_{n+1}\right)^{\prime}$. Since the ideal $\mathcal{L}$ is $\pi$-represented, we have the following chain of canonical isomorphisms:

$$
\begin{aligned}
\mathcal{M}\left(E_{1}, \ldots, E_{n} ; F^{\prime}\right) & \longleftrightarrow\left(E_{1} \otimes \cdots \otimes E_{n} \otimes F, \beta_{n+1}\right)^{\prime} \\
& \longleftrightarrow\left(E_{1} \otimes \cdots \otimes E_{n} \otimes F, \pi_{n+1}\right)^{\prime} \\
& \longleftrightarrow \mathcal{L}\left(E_{1}, \ldots, E_{n} ; F^{\prime}\right) .
\end{aligned}
$$

It follows that the identity operator is an algebraic isomorphism between the spaces $\mathcal{M}\left(E_{1}, \ldots, E_{n} ; F^{\prime}\right)$ and $\mathcal{L}\left(E_{1}, \ldots, E_{n} ; F^{\prime}\right)$, which is a contradiction. 
The next multilinear generalization of the ideal of absolutely summing linear operators was introduced in [5].

Definition 2.10. Given $p \geq q \geq 1$, a multilinear mapping $T \in \mathcal{L}\left(E_{1}, \ldots, E_{n} ; F\right)$ is said to be strongly multiple $(p, q)$-summing if there exists $C \geq 0$ such that

$$
\begin{aligned}
& \left(\sum_{j_{1}, \ldots, j_{n}=1}^{m}\left\|T\left(x_{j_{1}}^{(1)}, \ldots, x_{j_{n}}^{(n)}\right)\right\|^{p}\right)^{1 / p} \\
& \quad \leq C \sup _{\phi \in B_{\mathcal{L}\left(E_{1}, \ldots, E_{n}\right)}}\left(\sum_{j_{1}, \ldots, j_{n}=1}^{m}\left|\phi\left(x_{j_{1}}^{(1)}, \ldots, x_{j_{n}}^{(n)}\right)\right|^{q}\right)^{1 / q}
\end{aligned}
$$

for all $m \in \mathbb{N}$ and $x_{j_{l}}^{(l)} \in E_{l}$ where $l=1, \ldots, n$ and $j_{l}=1, \ldots, m$. The space of all strongly multiple $(p, q)$-summing $n$-linear mappings from $E_{1} \times \cdots \times E_{n}$ to $F$ will be denoted by $\mathcal{L}_{\mathrm{sm}(p, q)}\left(E_{1}, \ldots, E_{n} ; F\right)$. The infimum of the constants $C$ for which the inequality always holds defines a complete norm $\|\cdot\|_{\mathrm{sm}(p, q)}$ on $\mathcal{L}_{\text {sm }(p, q)}\left(E_{1}, \ldots, E_{n} ; F\right)$. When $p=q$ we abbreviate to $\mathcal{L}_{\mathrm{sm}(p)}\left(E_{1}, \ldots, E_{n} ; F\right)$ and $\|\cdot\|_{\mathrm{sm}(p)}$.

PROPOSITION 2.11. The multi-ideal $\mathcal{L}_{\mathrm{sm}(p)}, \quad 1 \leq p<+\infty$, of strongly multiple p-summing multilinear mappings is represented by a tensor norm but not by a smooth tensor norm.

PROOF. Following the lines of [21, Proposición 4.37], it is not difficult to prove that $\mathcal{L}_{\mathrm{sm}(p)}$ is maximal. It follows from [16, Theorem 4.5] that $\mathcal{L}_{\mathrm{sm}(p)}$ is represented by a tensor norm.

Let us see that $\mathcal{L}_{\mathrm{sm}(p)}$ cannot be represented by a smooth tensor norm. It is clear that

$$
\mathcal{L}_{\mathrm{sm}(p)}\left(E_{1}, \ldots, E_{n}, F ; \mathbb{K}\right)=\mathcal{L}\left(E_{1}, \ldots, E_{n}, F ; \mathbb{K}\right)
$$

for all integers $n$ and Banach spaces $E_{1}, \ldots, E_{n}$ and $F$. On the other hand, if we assumed that $\mathcal{L}_{\mathrm{sm}(p)}\left({ }^{n} E ; F^{\prime}\right)=\mathcal{L}\left({ }^{n} E ; F^{\prime}\right)$ for all integers $n$ and Banach spaces $E$ and $F$, by [5, Proposition 5.2(iii)], then we would have $\mathcal{L}\left(E ; F^{\prime}\right)=\Pi_{p}\left(E ; F^{\prime}\right)$ for all $E$ and $F$. This is absurd because the canonical injection $E \hookrightarrow E^{\prime \prime}=\left(E^{\prime}\right)^{\prime}$ fails to be $p$-summing for every infinite-dimensional Banach space $E$. Hence $\mathcal{L}\left({ }^{n} E ; F^{\prime}\right) \neq$ $\mathcal{L}_{\text {sm }(p)}\left({ }^{n} E ; F^{\prime}\right)$ for some $n$ and some Banach spaces $E$ and $F$. By Proposition 2.9 it follows that $\mathcal{L}_{\mathrm{sm}(p)}$ cannot be represented by a smooth tensor norm.

Corollary 2.12. If $\mathcal{M}=\mathcal{L}_{\mathrm{sm}(p)}$, then $\mathcal{M} \neq \mathcal{L}_{\beta} \mathcal{M}$.

PROOF. It is easy to check that $\mathcal{L}_{\mathrm{sm}(p)}$ has property [B]. Indeed, it is enough to combine the definition of $\mathcal{L}_{\mathrm{sm}(p)}$ with the well-known fact that the space of $(n+1)$ linear forms $\mathcal{L}\left(E_{1}, \ldots, E_{n}, \mathbb{K} ; \mathbb{K}\right)$ is isometrically isomorphic to the space of $n$-linear forms $\mathcal{L}\left(E_{1}, \ldots, E_{n} ; \mathbb{K}\right)$ via the obvious correspondence. Assuming that $\mathcal{M}=\mathcal{L}_{\beta} \mathcal{M}$, $\mathcal{L}_{\mathrm{sm}(p)}$ would be represented by a smooth tensor norm by Proposition 2.7; but this is not true by Proposition 2.11. 
REMARK 2.13. For the sake of completeness, we construct the tensor norm that represents the multi-ideal $\mathcal{L}_{\mathrm{sm}(p)}$. Given normed spaces $E_{1}, \ldots, E_{n}, F$ and $p \geq 1$, we define $\beta_{p}(u)$ to be the infimum of all expressions

$$
\sum_{m=1}^{M}\left\|\left(b_{m, j_{m}^{1}, \ldots, j_{m}^{n}}\right){ }_{j_{m}^{1}, \ldots, j_{m}^{n}=1}^{I_{m}^{1}, \ldots, I_{m}^{n}}\right\|_{q} \sup _{\phi \in B_{\mathcal{L}\left(E_{1}, \ldots, E_{n}\right)}}\left(\sum_{j_{m}^{1}, \ldots, j_{m}^{n}=1}^{I_{m}^{1}, \ldots, I_{m}^{n}} \mid \phi\left(x_{m, j_{m}^{1}}^{(1)}, \ldots,\left.x_{m, j_{m}^{n}}^{(n)}\right|^{p}\right)^{1 / p},\right.
$$

over all representations of the tensor $u \in E_{1} \otimes \cdots \otimes E_{n} \otimes F$ of the form

$$
u=\sum_{m=1}^{M} \sum_{j_{m}^{1}, \ldots, j_{m}^{n}=1}^{I_{m}^{1}, \ldots, I_{m}^{n}} x_{m, j_{m}^{1}}^{(1)} \otimes \cdots \otimes x_{m, j_{m}^{n}}^{(n)} \otimes b_{m, j_{m}^{1}, \ldots, j_{m}^{n}}
$$

where $M \in \mathbb{N}, x_{m, j_{m}^{l}}^{(l)} \in E_{l}$ when $l=1, \ldots, n, b_{m, j_{m}^{1}, \ldots, j_{m}^{n}} \in F, j_{m}^{l}=1, \ldots, I_{m}^{l}, q \geq 1$ and $1 / p+1 / q=1$. Following the lines of [21, Teorema 4.38], one can prove that $\beta_{p}$ is a tensor norm and that $\mathcal{L}_{\mathrm{sm}(p)}$ is $\beta_{p}$-represented. From Proposition 2.11 it follows that $\beta_{p}$ is not smooth.

The ideal $\mathcal{L}_{\mathrm{m}(p)}$ of multiple $p$-summing multilinear mappings, introduced by Matos [20] and, independently, by Bombal et al. [2], has played a central role in the theory of multi-ideals, even providing unexpected applications (see Remark 2.15).

PROpOsition 2.14. If $1 \leq p \leq 2$, then the ideal $\mathcal{L}_{\mathrm{m}(p)}$ of multiple p-summing multilinear mappings is represented by a tensor norm but not by a smooth tensor norm.

PROOF. It is proved in [21, Proposición 4.39] that $\mathcal{L}_{\mathrm{m}(p)}$ is represented by a tensor norm. Since $\mathcal{L}_{\mathrm{m}(p)}\left({ }^{2} \ell_{1} ; \mathbb{K}\right)=\mathcal{L}\left({ }^{2} \ell_{1} ; \mathbb{K}\right)$ (see [21, Teorema 5.23]) and

$$
\Pi_{p}\left(\ell_{1} ; \ell_{\infty}\right)=\mathcal{L}_{\mathrm{m}(p)}\left(\ell_{1} ; \ell_{\infty}\right) \neq \mathcal{L}\left(\ell_{1} ; \ell_{\infty}\right)
$$

(obvious), the nonrepresentability by a smooth tensor norm follows from Proposition 2.9.

REMARK 2.15. As proved by Defant and Pérez-García [12], the tensor norm that represents the ideal $\mathcal{L}_{\mathrm{m}}(p)$ of multiple $p$-summing multilinear mappings is the first example of a tensor norm that preserves unconditionality for $\mathcal{L}_{p}$-spaces.

REMARK 2.16. Everything that we proved for the multi-ideal $\mathcal{L}_{\mathrm{sm}(p)}$ of strongly multiple $p$-summing multilinear mappings can also be proved, mutatis mutandis, for the multi-ideal $\mathcal{L}_{\mathrm{ss}(p)}$ of strongly $p$-summing multilinear mappings introduced in [15].

We finish this section with another hint that the representation of a (vector-valued) multi-ideal by a smooth tensor norm is unusual.

PROPOSITION 2.17. The multi-ideal $\mathcal{L}_{\varepsilon}$ is not represented by a smooth tensor norm.

PROOF. Assume that $\mathcal{L}_{\varepsilon}$ is represented by a smooth tensor norm $\beta=\left(\beta_{n}\right)_{n=1}^{\infty}$. Fix a positive integer $n$ and Banach spaces $E_{1}, \ldots, E_{n}$. Since $\mathcal{L}_{\varepsilon}$ is $\beta$-represented, 
the mapping

$$
\begin{gathered}
\varphi:\left(\mathcal{L}_{\varepsilon}\left(E_{1}, \ldots, E_{n} ; F^{\prime}\right),\|\cdot\|_{\mathcal{L}_{\varepsilon}}\right) \longrightarrow\left(E_{1} \otimes \cdots \otimes E_{n} \otimes F, \beta_{n+1}\right)^{\prime} \\
T \longrightarrow \varphi(T)\left(x_{1} \otimes \cdots \otimes x_{n} \otimes y\right)=T\left(x_{1}, \ldots, x_{n}\right)(y)
\end{gathered}
$$

is an isometric isomorphism for all $F$. In particular, taking $F=\mathbb{K}$, we obtain the following isometric isomorphisms:

$$
\begin{aligned}
\left(\mathcal{L}_{\varepsilon}\left(E_{1}, \ldots, E_{n} ; \mathbb{K}^{\prime}\right),\|\cdot\|_{\mathcal{L}_{\varepsilon}}\right) & \cong\left(E_{1} \otimes \cdots \otimes E_{n} \otimes \mathbb{K}, \beta_{n+1}\right)^{\prime} \\
& \cong\left(E_{1} \otimes \cdots \otimes E_{n}, \beta_{n}\right)^{\prime} .
\end{aligned}
$$

In Corollary 3.2, we prove that the following spaces are also isometrically isomorphic, with the same canonical correspondences:

$$
\begin{aligned}
\left(\mathcal{L}_{\varepsilon}\left(E_{1}, \ldots, E_{n} ; \mathbb{K}^{\prime}\right),\|\cdot\|_{\mathcal{L}_{\varepsilon}}\right) & \cong\left(E_{1} \otimes \cdots \otimes E_{n} \otimes \mathbb{K}, \varepsilon_{n+1}\right)^{\prime} \\
& \cong\left(E_{1} \otimes \cdots \otimes E_{n}, \varepsilon_{n}\right)^{\prime} .
\end{aligned}
$$

Hence the identity mapping

$$
\left(E_{1} \otimes \cdots \otimes E_{n}, \varepsilon_{n}\right)^{\prime} \longrightarrow\left(E_{1} \otimes \cdots \otimes E_{n}, \beta_{n}\right)^{\prime}
$$

is an isometric isomorphism. Calling on the Hahn-Banach theorem once again, we see that $\varepsilon_{n}$ and $\beta_{n}$ coincide on $E_{1} \otimes \cdots \otimes E_{n}$. It follows that the ideal $\mathcal{L}_{\varepsilon}$ is $\varepsilon$-represented, which contradicts Proposition 2.8.

\section{The scalar-valued case}

The aim of this section is to show that smooth tensor norms are more suitable to represent ideals of multilinear forms.

Given a tensor norm $\beta$, we write $\mathcal{L}_{\beta}^{\mathbb{K}}:=\left(\mathcal{L}_{\beta}\right)^{\mathbb{K}}$.

We shall write $\mathcal{M}^{\mathbb{K}} \stackrel{1}{=} \mathcal{L}_{\beta}^{\mathbb{K}}$ if, for all $n$ and $E_{1}, \ldots, E_{n}$, the linearization operator $\Phi=\Phi\left(n, E_{1}, \ldots, E_{n}\right)$, given by

$$
A \in \mathcal{M}\left(E_{1}, \ldots, E_{n} ; \mathbb{K}\right) \mapsto \Phi(A):=A_{L},
$$

is an isometric isomorphism from $\mathcal{M}\left(E_{1}, \ldots, E_{n} ; \mathbb{K}\right)$ onto $\left(E_{1} \otimes \cdots \otimes E_{n}, \beta_{n}\right)^{\prime}$.

THEOREM 3.1. Let $\mathcal{M}$ be a multi-ideal and $\beta=\left(\beta_{n}\right)_{n=1}^{\infty}$ be a smooth tensor norm. Then the ideal of multilinear forms $\mathcal{M}^{\mathbb{K}}$ is $\beta$-represented if and only if $\mathcal{M}^{\mathbb{K}} \stackrel{1}{=} \mathcal{L}_{\beta}^{\mathbb{K}}$.

PRoOF. Let $n \in \mathbb{N}$ and $E_{1}, \ldots, E_{n}$ be Banach spaces. We shall say that $\Phi$ is well defined, isometric and onto if

$$
\Phi(A)=A_{L} \in\left(E_{1} \otimes \cdots \otimes E_{n}, \beta_{n}\right)^{\prime}
$$

for all $A \in \mathcal{M}\left(E_{1}, \ldots, E_{n} ; \mathbb{K}\right)$ and

$$
\Phi: \mathcal{M}\left(E_{1}, \ldots, E_{n} ; \mathbb{K}\right) \longrightarrow\left(E_{1} \otimes \cdots \otimes E_{n}, \beta_{n}\right)^{\prime}
$$

is isometric and surjective. Assume that $\Phi$ is well defined, isometric and onto. It is clear that $\Phi$ is linear and injective, so $\Phi$ is well defined, isometric and onto if and only if $\Phi$ is an isometric isomorphism from $\mathcal{M}\left(E_{1}, \ldots, E_{n} ; \mathbb{K}\right)$ onto $\left(E_{1} \otimes \cdots \otimes E_{n} \beta_{n}\right)^{\prime}$. 
We continue assuming that $\Phi$ is well defined, isometric and onto. Define the isometric isomorphism $h: \mathbb{K}^{\prime} \longrightarrow \mathbb{K}$ by $h(f)=f(1)$ for all $f \in \mathbb{K}^{\prime}$. It is clear that the linear mapping

$$
\xi: \mathcal{M}\left(E_{1}, \ldots, E_{n} ; \mathbb{K}^{\prime}\right) \longrightarrow \mathcal{M}\left(E_{1}, \ldots, E_{n} ; \mathbb{K}\right), \quad \xi(A)=h \circ A,
$$

is an isometric isomorphism as well. Considering the chain

$$
\begin{aligned}
\mathcal{M}\left(E_{1}, \ldots, E_{n} ; \mathbb{K}^{\prime}\right) \stackrel{\xi}{\longrightarrow} & \mathcal{M}\left(E_{1}, \ldots, E_{n} ; \mathbb{K}\right) \stackrel{\Phi}{\longrightarrow}\left(E_{1} \otimes \cdots \otimes E_{n}, \beta_{n}\right)^{\prime} \\
\stackrel{\psi^{*}}{\longrightarrow} & \left(E_{1} \otimes \cdots \otimes E_{n} \otimes \mathbb{K}, \beta_{n+1}\right)^{\prime},
\end{aligned}
$$

where $\psi^{*}$ is the adjoint of the linear operator $\psi$ of the definition of smooth tensor norm, it is not difficult to check that $\varphi=\psi^{*} \circ \Phi \circ \xi$ (hence $\Phi=\left(\psi^{*}\right)^{-1} \circ \varphi \circ \xi^{-1}$ ), where $\varphi=\varphi\left(n, E_{1}, \ldots, E_{n}\right)$ is the operator of Definition 1.3.

Hence the following are equivalent:

(a) $\mathcal{M}^{\mathbb{K}} \stackrel{1}{=} \mathcal{L}_{\beta}^{\mathbb{K}}$

(b) $\Phi\left(n, E_{1}, \ldots, E_{n}\right)$ is an isometric isomorphism for all $n, E_{1}, \ldots, E_{n}$;

(c) $\Phi\left(n, E_{1}, \ldots, E_{n}\right)$ is well defined, isometric and onto for all $n, E_{1}, \ldots, E_{n}$;

(d) $\varphi\left(n, E_{1}, \ldots, E_{n}\right)$ is an isometric isomorphism for all $n, E_{1}, \ldots, E_{n}$;

(e) $\mathcal{M}^{\mathbb{K}}$ is $\beta$-represented.

The proof is thus complete.

As we saw before (for example, Proposition 2.8 and Corollary 2.12), the theorem above cannot be generalized to vector-valued multi-ideals.

COROLLARY 3.2. Let $\beta$ be a smooth tensor norm. Then the ideal of multilinear forms $\mathcal{L}_{\beta}^{\mathbb{K}}$ is $\beta$-represented and thus is represented by a smooth tensor norm.

EXAMPLE 3.3. The ideal of multilinear forms $\mathcal{L}_{\varepsilon}^{\mathbb{K}}$ is $\varepsilon$-represented, hence represented by a smooth tensor norm.

COROLlary 3.4. Let $\mathcal{M}$ be a multi-ideal. If $\mathcal{M}^{\mathbb{K}}$ is represented by a smooth tensor norm then $\mathcal{M}^{\mathbb{K}}$ contains the integral multilinear forms.

PROOF. By Theorem 3.1 we have $\mathcal{M}^{\mathbb{K}}=\mathcal{L}_{\beta}^{\mathbb{K}}$, where $\beta$ is a smooth tensor norm. Since $\varepsilon \leq \beta$ because $\beta$ is a tensor norm, it follows that

$$
\mathcal{L}_{\mathcal{I}}^{\mathbb{K}}=\mathcal{L}_{\varepsilon}^{\mathbb{K}} \subseteq \mathcal{L}_{\beta}^{\mathbb{K}}=\mathcal{M}^{\mathbb{K}}
$$

as required.

Next we see that sometimes we can construct explicitly the smooth tensor norm that represents an ideal of multilinear forms.

Definition 3.5. Let $p \geq 1$. An $n$-linear mapping $A \in \mathcal{L}\left(E_{1}, \ldots, E_{n} ; F\right)$ is p-semi-integral, written $A \in \mathcal{L}_{\mathrm{si}(p)}\left(E_{1}, \ldots, E_{n} ; F\right)$, if there exist $C \geq 0$ and a regular probability measure $\mu$ on the Borel $\sigma$-algebra of $B_{E_{1}^{\prime}} \times \cdots \times B_{E_{n}^{\prime}}$, endowed with the 
product of the weak-star topologies $\sigma\left(E_{l}^{\prime}, E_{l}\right)$ where $l=1, \ldots, n$, such that

$$
\left\|A\left(x_{1}, \ldots, x_{n}\right)\right\| \leq C\left(\int_{B_{E_{1}^{\prime}} \times \cdots \times B_{E_{n}^{\prime}}}\left|\varphi_{1}\left(x_{1}\right) \cdots \varphi_{n}\left(x_{n}\right)\right|^{p} d \mu\left(\varphi_{1}, \ldots, \varphi_{n}\right)\right)^{1 / p}
$$

for all $x_{j} \in E_{j}$, where $j=1, \ldots, n$. The infimum of the constants $C$ defines a norm $\|\cdot\|_{\mathrm{si}(p)}$ on $\mathcal{L}_{\mathrm{si}(p)}\left(E_{1}, \ldots, E_{n} ; F\right)$.

It is well known that $\mathcal{L}_{\mathrm{si}(p)}$ is a multi-ideal (see [7]).

PROPOSITION 3.6. When $p \geq 1$, the ideal of $p$-semi-integral multilinear forms $\mathcal{L}_{\mathrm{si}(p)}^{\mathbb{K}}$ is represented by a smooth tensor norm.

Proof. Given $u \in E_{1} \otimes \cdots \otimes E_{n}$, define

$$
\sigma_{p}^{n}(u):=\inf \left\|\left(\lambda_{j}\right)_{j=1}^{m}\right\|_{q}\left(\sup _{\varphi_{l} \in B_{E_{l}^{\prime}}} \sum_{j=1}^{m}\left|\varphi_{1}\left(x_{1, j}\right) \cdots \varphi_{n}\left(x_{n, j}\right)\right|^{p}\right)^{1 / p}
$$

where the infimum is taken over all representations of the form

$$
u=\sum_{j=1}^{m} \lambda_{j} x_{1, j} \otimes \cdots \otimes x_{n, j}
$$

where $m \in \mathbb{N}, x_{l, j} \in E_{l}$ when $l=1, \ldots, n, \lambda_{j} \in \mathbb{K}$ when $j=1, \ldots, m$, and $1 / p+$ $1 / q=1$. Standard techniques (and hard work) show that $\sigma_{p}=\left(\sigma_{p}^{n}\right)_{n=1}^{\infty}$ is a tensor norm. Let us show that $\sigma_{p}$ is smooth. Given

$$
u=\sum_{j=1}^{m} \lambda_{j} x_{1, j} \otimes \cdots \otimes x_{n, j} \otimes b_{j} \in E_{1} \otimes \cdots \otimes E_{n} \otimes \mathbb{K},
$$

we have

$$
\begin{aligned}
\left(\sigma_{p}^{n+1}(u)\right)^{p} & \leq\left\|\left(\lambda_{j}\right)_{j=1}^{m}\right\|_{q}^{p} \sup _{\varphi l \in B_{E_{l}^{\prime}}, \varphi \in B_{\mathbb{K}^{\prime}}} \sum_{j=1}^{m}\left|\varphi_{1}\left(x_{1, j}\right) \cdots \varphi_{n}\left(x_{n, j}\right) \varphi\left(b_{j}\right)\right|^{p} \\
& =\left\|\left(\lambda_{j}\right)_{j=1}^{m}\right\|_{q}^{p} \sup _{\varphi_{l} \in B_{E_{l}^{\prime}}, \varphi \in B_{\mathbb{K}^{\prime}}} \sum_{j=1}^{m}\left|\varphi_{1}\left(b_{j} x_{1, j}\right) \cdots \varphi_{n}\left(x_{n, j}\right) \varphi(1)\right|^{p} \\
& \leq\left\|\left(\lambda_{j}\right)_{j=1}^{m}\right\|_{q}^{p} \sup _{\varphi_{l} \in B_{E_{l}^{\prime}}, \varphi \in B_{\mathbb{K}^{\prime}}} \sum_{j=1}^{m}\left|\varphi_{1}\left(b_{j} x_{1, j}\right) \cdots \varphi_{n}\left(x_{n, j}\right)\right|^{p}\|\varphi\|^{p}|1|^{p} \\
& \leq\left\|\left(\lambda_{j}\right)_{j=1}^{m}\right\|_{q}^{p} \sup _{\varphi_{l} \in B_{E_{l}^{\prime}}} \sum_{j=1}^{m}\left|\varphi_{1}\left(b_{j} x_{1, j}\right) \cdots \varphi_{n}\left(x_{n, j}\right)\right|^{p} .
\end{aligned}
$$

Since $\sum_{j=1}^{m} \lambda_{j}\left(b_{j} x_{1, j}\right) \otimes \cdots \otimes x_{n, j}$ is a representation of $\psi(u) \in E_{1} \otimes \cdots \otimes E_{n}$ and each representation of $\psi(u)$ there corresponds to a representation of $u$, it follows that $\sigma_{p}^{n+1}(u) \leq \sigma_{p}^{n}(\psi(u))$. A similar computation shows that $\sigma_{p}^{n}(\psi(u)) \leq \sigma_{p}^{n+1}(u)$, and completes the proof of the smoothness of $\sigma_{p}$. 
The proof that $\mathcal{L}_{\mathrm{si}(p)}^{\mathbb{K}}$ is $\sigma_{p}$-represented is a combination of the arguments of the proofs of [1, Theorem 4.8] and [7, Theorem 1]. Take $E_{1}, \ldots, E_{n}$ and $f \in$ $\left(E_{1} \otimes \cdots \otimes E_{n}, \sigma_{p}^{n}\right)^{\prime}$, and define the $n$-linear form $A$ on $E_{1} \times \cdots \times E_{n}$ by

$$
A\left(x_{1}, \ldots, x_{n}\right)=f\left(x_{1} \otimes \cdots \otimes x_{n}\right) .
$$

For appropriate $\lambda_{j} \in \mathbb{K}$ such that $\left|\lambda_{j}\right|=1$ when $j=1, \ldots, m$,

$$
\begin{aligned}
\sum_{j=1}^{m} \mid & \left.A\left(x_{1, j}, \ldots, x_{n, j}\right)\right|^{p} \\
= & \left.\sum_{j=1}^{m}|| f\left(x_{1, j} \otimes \cdots \otimes x_{n, j}\right)\right|^{p-1} f\left(x_{1, j} \otimes \cdots \otimes x_{n, j}\right) \mid \\
= & \left.\left|\sum_{j=1}^{m}\right| f\left(x_{1, j} \otimes \cdots \otimes x_{n, j}\right)\right|^{p-1} \lambda_{j} f\left(x_{1, j} \otimes \cdots \otimes x_{n, j}\right) \mid \\
= & \left|f\left(\sum_{j=1}^{m} \lambda_{j}\left|f\left(x_{1, j} \otimes \cdots \otimes x_{n, j}\right)\right|^{p-1} x_{1, j} \otimes \cdots \otimes x_{n, j}\right)\right| \\
\leq & \|f\|_{\left(E_{1} \otimes \cdots \otimes E_{n}, \sigma_{p}^{n}\right)^{\prime}} \sigma_{p}^{n} \\
& \times\left(\sum_{j=1}^{m} \lambda_{j}\left|f\left(x_{1, j} \otimes \cdots \otimes x_{n, j}\right)\right|^{p-1} x_{1, j} \otimes \cdots \otimes x_{n, j}\right) \\
\leq & \|f\|_{\left(E_{1} \otimes \cdots \otimes E_{n}, \sigma_{p}^{n}\right)^{\prime}}\left\|\left(\lambda_{j}\left|f\left(x_{1, j} \otimes \cdots \otimes x_{n, j}\right)\right|^{p-1}\right)_{j=1}^{m}\right\|_{q} \\
& \times\left(\sup _{\varphi_{l} \in B_{E_{l}^{\prime}}} \sum_{j=1}^{m}\left|\varphi_{1}\left(x_{1, j}\right) \cdots \varphi_{n}\left(x_{n, j}\right)\right|^{p}\right)^{1 / p} \\
= & \|f\|_{\left(E_{1} \otimes \cdots \otimes E_{n}, \sigma_{p}^{n}\right)^{\prime}}\left(\sum_{j=1}^{m}\left|f\left(x_{1, j} \otimes \cdots \otimes x_{n, j}\right)\right|^{p}\right)^{1 / q} \\
& \times\left(\sup _{\varphi_{l} \in B_{E_{l}^{\prime}}} \sum_{j=1}^{m}\left|\varphi_{1}\left(x_{1, j}\right) \cdots \varphi_{n}\left(x_{n, j}\right)\right|^{p}\right)^{1 / p} \cdot
\end{aligned}
$$

Hence

$$
\begin{aligned}
& \left(\sum_{j=1}^{m}\left|A\left(x_{1, j}, \ldots, x_{n, j}\right)\right|^{p}\right)^{1 / p} \\
& \quad \leq\|f\|_{\left(E_{1} \otimes \cdots \otimes E_{n}, \sigma_{p}^{n}\right)^{\prime}}\left(\sup _{\varphi_{l} \in B_{E_{l}^{\prime}}} \sum_{j=1}^{m}\left|\varphi_{1}\left(x_{1, j}\right) \cdots \varphi_{n}\left(x_{n, j}\right)\right|^{p}\right)^{1 / p},
\end{aligned}
$$

which shows that $A$ is $p$-semi-integral and

$$
\|A\|_{\mathrm{si}(p)} \leq\|f\|_{\left(E_{1} \otimes \cdots \otimes E_{n}, \sigma_{p}^{n}\right)^{\prime}}=\|\Phi(A)\|_{\left(E_{1} \otimes \cdots \otimes E_{n}, \sigma_{p}^{n}\right)^{\prime}} .
$$


In particular, $\Phi$ is surjective. In order to establish the reverse inequality, we take $A \in \mathcal{L}_{\text {si }(p)}\left(E_{1}, \ldots, E_{n} ; \mathbb{K}\right)$ and let

$$
u=\sum_{j=1}^{m} \lambda_{j} x_{1, j} \otimes \cdots \otimes x_{n, j} \in E_{1} \otimes \cdots \otimes E_{n},
$$

where $\lambda_{j} \in \mathbb{K}, x_{l, j} \in E_{l}, l=1, \ldots, n$, and $j=1, \ldots, m$. From [7, Theorem 1], it follows that

$$
\begin{aligned}
|\Phi(A)(u)|^{p} & =\left|\sum_{j=1}^{m} \lambda_{j} A\left(x_{1, j}, \ldots, x_{n, j}\right)\right|^{p} \\
& \leq\left\|\left(\lambda_{j}\right)_{j=1}^{m}\right\|_{q}^{p} \sum_{j=1}^{m}\left|A\left(x_{1, j}, \ldots, x_{n, j}\right)\right|^{p} \\
& \leq\left\|\left(\lambda_{j}\right)_{j=1}^{m}\right\|_{q}^{p}\|A\|_{\mathrm{si}(p)}^{p} \sup _{\varphi_{l} \in B_{E_{l}^{\prime}}} \sum_{j=1}^{m}\left|\varphi_{1}\left(x_{1, j}\right) \cdots \varphi_{n}\left(x_{n, j}\right)\right|^{p},
\end{aligned}
$$

so $|\Phi(A)(u)| \leq\|A\|_{\operatorname{si}(p)} \cdot \sigma_{p}(u)$. Since $u$ is arbitrary it follows that

$$
\|\Phi(A)\|_{\left(E_{1} \otimes \cdots \otimes E_{n}, \sigma_{p}\right)^{\prime}} \leq\|A\|_{\mathrm{si}(p)},
$$

which completes the proof.

Summing up, we conclude the following. First, the multi-ideal $\mathcal{L}_{\varepsilon}$ is not represented by a smooth tensor norm (Proposition 2.17), whereas $\mathcal{L}_{\varepsilon}^{\mathbb{K}}$ is represented by the smooth tensor norm $\varepsilon$ (Example 3.3). Next, the ideal $\mathcal{L}_{\mathrm{sm}(p)}$ of strongly multiple $p$-summing multilinear mappings is represented by a tensor norm but not by a smooth tensor norm (Proposition 2.11). As in Proposition 3.6, it can be proved that $\mathcal{L}_{\mathrm{sm}(p)}^{\mathbb{K}}$ is represented by a smooth tensor norm. Third, the ideal $\mathcal{L}_{\mathrm{si}(p)}^{\mathbb{K}}$ of $p$-semi-integral multilinear forms is represented by a smooth tensor norm (Proposition 3.6), and the multi-ideal $\mathcal{L}_{\mathrm{si}(p)}$ of $p$-semi-integral multilinear mappings is represented by a tensor norm [6, Proposition 6] but not by a smooth tensor norm (this is hard work). Finally, it seems that, typically, the behavior of a maximal multiideal $\mathcal{M} \neq \mathcal{L}$ is that $\mathcal{M}$ is not represented by a smooth tensor norm whereas $\mathcal{M}^{\mathbb{K}}$ is represented by a smooth tensor norm.

We conclude this paper by showing how the representation of the ideal of multilinear functionals $\mathcal{M}^{\mathbb{K}}$ by a smooth tensor norm provides information about the representation of certain vector-valued components of $\mathcal{M}$. Given Banach spaces $E_{1}, \ldots, E_{n}, F$ and a tensor norm $\beta=\left(\beta_{n}\right)_{n=1}^{\infty}$, we shall say that $\mathcal{M}\left(E_{1}, \ldots, E_{n} ; F^{\prime}\right)$ is $\beta$-represented if $\mathcal{M}\left(E_{1}, \ldots, E_{n} ; F^{\prime}\right)$ and $\left(E_{1} \otimes \cdots \otimes E_{n} \otimes\right.$ $\left.F, \beta_{n+1}\right)^{\prime}$ are isometrically isomorphic via the canonical mapping of Definition 1.3.

Proposition 3.7. Let $\mathcal{M}$ be a multi-ideal such that $\mathcal{M}^{\mathbb{K}}$ is represented by a smooth tensor norm $\beta$. Then $\mathcal{M}\left(E_{1}, \ldots, E_{n} ; F^{\prime}\right)$ is $\beta$-represented whenever the spaces $\mathcal{M}\left(E_{1}, \ldots, E_{n} ; F^{\prime}\right)$ and $\mathcal{M}\left(E_{1}, \ldots, E_{n}, F ; \mathbb{K}\right)$ are canonically isometrically isomorphic. 
PROOF. Consider the following chain of canonical mappings:

$$
\begin{aligned}
\mathcal{M}\left(E_{1}, \ldots, E_{n} ; F^{\prime}\right) & =\mathcal{M}\left(E_{1}, \ldots, E_{n}, F ; \mathbb{K}\right) \\
& =\mathcal{M}\left(E_{1}, \ldots, E_{n}, F ; \mathbb{K}^{\prime}\right) \\
& =\left(E_{1} \otimes \cdots \otimes E_{n} \otimes F \otimes \mathbb{K}, \beta_{n+2}\right)^{\prime} \\
& =\left(E_{1} \otimes \cdots \otimes E_{n} \otimes F, \beta_{n+1}\right)^{\prime}
\end{aligned}
$$

The first equality is an isometric isomorphism by assumption; the second equality holds because the canonical mapping

$$
h: \mathbb{K} \longrightarrow \mathbb{K}^{\prime}, \quad h(\lambda)(\alpha)=\lambda \cdot \alpha
$$

is an isometric isomorphism; the third equality is because $\mathcal{M}^{\mathbb{K}}$ is $\beta$-represented; and the last equality holds because the tensor norm $\beta$ is smooth. Routine computations show that the composition of all these mappings yields the canonical mapping from $\mathcal{M}\left(E_{1}, \ldots, E_{n} ; F^{\prime}\right)$ to $\left(E_{1} \otimes \cdots \otimes E_{n} \otimes F, \beta_{n+1}\right)^{\prime}$.

\section{Acknowledgements}

The authors thank the anonymous referee and the editor for their suggestions that substantially improved the presentation of the paper.

\section{References}

[1] R. Alencar and M. C. Matos, Some Classes of Multilinear Mappings between Banach Spaces, Publicaciones del Departamento de Análisis Matemático, 12 (Universidad Complutense de Madrid, Madrid, 1989).

[2] F. Bombal, D. Peréz-García and I. Villanueva, 'Multilinear extensions of Grothendieck's theorem', Q. J. Math. 55 (2004), 441-450.

[3] G. Botelho, H.-A. Braunss, H. Junek and D. Pellegrino, 'Holomorphy types and ideals of multilinear mappings', Studia Math. 177 (2006), 43-65.

[4] G. Botelho and D. Pellegrino, 'Two new properties of ideals of polynomials and applications', Indag. Math. (N.S.) 16 (2005), 157-169.

[5] G. Botelho and D. Pellegrino, 'Coincidence situations for absolutely summing nonlinear mappings', Port. Math. 64 (2007), 175-191.

[6] E. Çalişkan, 'On characterizations of the space of $p$-semi-integral multilinear mappings', Note Mat. 29 (2009), 165-184.

[7] E. Çalişkan and D. M. Pellegrino, 'On the multilinear generalizations of the concept of absolutely summing operators', Rocky Mountain J. Math. 37 (2007), 1137-1154.

[8] D. Carando, V. Dimant and S. Muro, 'Coherent sequences of polynomials ideals on Banach spaces', Math. Nachr. 282 (2009), 1111-1133.

[9] R. Cilia, M. D'Anna and J. Gutiérrez, 'Polynomial characterization of $\mathcal{L}_{\infty}$-spaces', J. Math. Anal. Appl. 275 (2002), 900-912.

[10] H. G. Dales, Banach Algebras and Automatic Continuity, London Mathematical Society Monographs New Series, 24 (Oxford University Press, Oxford, 2000).

[11] A. Defant and K. Floret, Tensor Norms and Operator Ideals, North-Holland Mathematical Studies, 176 (North-Holland, Amsterdam, 1993). 
[12] A. Defant and D. Pérez-García, 'A tensor norm preserving unconditionality for $L_{p}$-spaces', Trans. Amer. Math. Soc. 360 (2008), 3287-3306.

[13] J. Diestel, J. H. Fourie and J. Swart, The Metric Theory of Tensor Products, Grothendieck's Résumé Revisited (American Mathematical Society, Providence, RI, 2008).

[14] J. Diestel, H. Jarchow and A. Tonge, Absolutely Summing Operators, Cambridge Studies in Advanced Mathematics, 43 (Cambridge University Press, Cambridge, 1995).

[15] V. Dimant, 'Strongly p-summing multilinear operators', J. Math. Anal. Appl. 278 (2003), 182-193.

[16] K. Floret and S. Hunfeld, 'Ultrastability of ideals of homogeneous polynomials and multilinear mappings on Banach spaces', Proc. Amer. Math. Soc. 130 (2002), 1425-1435.

[17] A. Grothendieck, 'Résumé de la théorie métrique des produits tensoriels topologiques', Bol. Soc. Mat. São Paulo 8 (1956), 1-79.

[18] J. Lindenstrauss and A. Pełczyński, 'Absolutely summing operators in $L_{p}$ spaces and their applications', Studia Math. 29 (1968), 275-326.

[19] M. C. Matos, 'On multilinear mappings of nuclear type', Rev. Mat. Univ. Complut. Madrid 6 (1993), 61-81.

[20] M. C. Matos, 'Fully absolutely summing and Hilbert-Schmidt multilinear mappings', Collect. Math. 54 (2003), 111-136.

[21] D. Pérez-García, 'Operadores multilineales absolutamente sumantes', PhD Thesis, Universidad Complutense de Madrid, Madrid, 2003.

[22] R. Ryan, Introduction to Tensor Products of Banach Spaces, Springer Monographs in Mathematics (Springer, London, 2002).

GERALDO BOTELHO, Faculdade de Matemática, Universidade Federal de Uberlândia, 38.400-902 Uberlândia, Brazil e-mail: botelho@ufu.br

ERHAN ÇALIŞKAN, Yıldız Teknik Üniversitesi, Fen-Edebiyat Fakültesi, Matematik Bölümü, Davutpaşa Kampüsü, 34210 Esenler, İstanbul, Türkíye e-mail: caliskan@yildiz.edu.tr

DANIEL PELLEGRINO, Departamento de Matemática, Universidade Federal da Paraíba, 58.051-900 João Pessoa, Brazil e-mail: dmpellegrino@gmail.com 